\title{
Life cycle assessment and cost analysis evaluation of a helicopter's canopy production using different manufacturing processes
}

\author{
Andreas Loukopoulos ${ }^{1}$, Christos Katsiropoulos*1 and Spiros Pantelakis ${ }^{1}$ \\ ${ }^{1}$ Laboratory of Technology \& Strength of Materials, Dept. of Mechanical Engineering \& Aeronautics, \\ University of Patras, Panepistimioupolis Rion, 26500 Patras, Greece
}

\begin{abstract}
In the present work, Life Cycle analysis (LCA) and Life cycle costing (LCC) models were developed in order to quantify the environmental footprint and cost and thus compare different manufacturing scenarios associated with the production of aeronautical structural components. To validate the models developed, they were implemented for the case of a helicopter's canopy processed by two techniques commonly used in aeronautics, namely the autoclave and the Resin Transfer moulding (RTM). The canopy was assumed to be made of a carbon fiber reinforced thermosetting material. Using the models developed the expected environmental and cost benefits by involving the RTM technique have been quantified.
\end{abstract}

\section{Introduction}

Carbon fiber reinforced thermosetting materials demonstrate superior specific properties and corrosion resistance as compared to traditional materials. These desirable properties have made composites a common material in aerospace applications thus leading to reduction of aircraft fuel consumption and green house gases.

Several studies have been conducted for evaluating the environmental and financial viability of structures made from composite materials. Timmis et al. [1] evaluated the environmental footprint reduction occurred from the adoption of composite materials in aviation. The results pointed out that although carbon fiber reinforced polymers demonstrate increased environmental impact during the manufacturing and disposal phase, they demonstrate a more environmentally friendly behaviour as compared to other traditional materials (aluminum) when the in use phase of the aircraft is taken into consideration. Furthermore, the increased environmental impact during the production of composite materials have made the development of both environmentally friendly and financially viable end of life treatment techniques essential. In this context, in [2] the environmental and financial credits of different waste treatments routes (disposal, incineration for energy recovery and mechanical recycling) of carbon fiber reinforced composites is investigated. The results demonstrated that the environmental benefits 
occurred from mechanical recycling are impaired from the severe deterioration of the mechanical properties of the fibers leading to a low market value product. In parallel, efforts have been undertaken in optimizing structural components with regard to their quality and cost [3],[4],[5]. In [3] the manufacturing of a helicopter's canopy produced using the cold diaphragm forming technique is optimized from a financial and qualitative standpoint. To this end, an optimization concept taking into account both the quality and the cost performance of a composite product was introduced and implemented. However, despite these efforts, tools and concepts allowing the simultaneous optimization of a product with regard to quality, cost and environmental impact are still not available.

The aim of this work is to contribute on developing a model for estimating the environmental footprint and cost occurring from the production of an aeronautical structural component. For this purpose for the same canopy optimized in [3] with regard to its cost and quality, Life Cycle analysis (LCA) and Life cycle costing models (LCC) are implemented. For developing these models the component is assumed to be made of a conventional carbon fiber reinforced thermosetting composite. As far as the manufacturing stage is concerned, two alternative processing routes commonly used in aeronautics, namely the conventional autoclave process and the Resin Transfer molding technique (RTM) were considered.

\section{Definition of the case study}

The present study explores alternative manufacturing and recycling scenarios in terms of financial and environmental viability, associated with the canopy of the EUROCOPTER EC Twin Star helicopter described in [3]. The canopy is assumed to be made of a carbon fiber reinforced epoxy composite material, with a $60 \%$ fiber volume fraction. The total mass of the canopy was calculated based on [3] and found equal to $0,64 \mathrm{~kg}$. As a result the mass of the carbon fibers and the epoxy were found equal to $0,384 \mathrm{~kg}$ and $0,256 \mathrm{~kg}$ respectively. The canopy taken for the present study has been assumed to be the same with the thermoplastic one shown in figure 1.

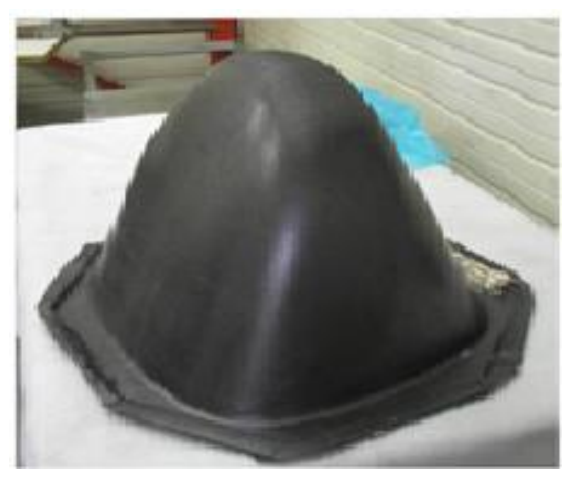

Figure 1:The canopy described in [3] (1:3 scale prototype of the real part). 


\section{Methodology}

\subsection{Life Cycle Analysis}

Life cycle analysis is a well established technique for assessing the environmental impact associated with a product or a process, from raw material extraction to disposal or recycling. The goal of this technique is to quantify all the energy and material inputs and outputs as well as the emissions to the environment associated with a process or a product so as to provide a sound basis for adopting environmental oriented decisions. In the present work, the ISO 14040:2006 was selected [6]. ISO 14040:2006 is a commonly used tool that describes the principles for carrying out an LCA. According to the above standard LCA involves four stages. The first stage is the goal and scope definition where the purpose of the study, the system boundaries and the level of detail are defined. The second stage of the LCA is the inventory analysis where all the inputs (raw materials, energy etc.) and outputs (emissions to the environment) of the system are quantified. The third stage of the LCA is the impact assessment which is a vital stage because it associates the inventory data with impact categories providing additional information for deeper understanding a product's environmental footprint. Finally, during the interpretation stage, the results of the analysis are summarized and discussed, as well as recommendations and decisions are made according to the initial goal of the analysis.

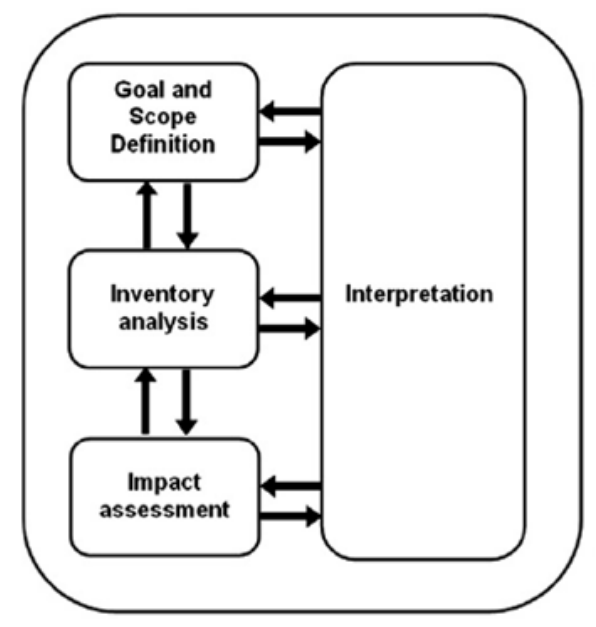

Figure 2: Outline of LCA according to ISO 14040:2006 [6].

Based on this approach, LCA models are developed for conducting a comparative analysis between different production and recycling scenarios of the canopy. The stages taken into consideration for the present LCA were: Carbon fiber production, Resin production, Manufacturing (autoclave and RTM), and Recycling (Mechanical recycling and pyrolysis).

For each of the abovementioned stages the total energy consumption as well as the Global Warming Potential-100 $\left(\mathrm{GWP}_{100}\right)$ for a period of 100 years were calculated. The 
$\mathrm{GWP}_{100}$ is a common index among LCA studies for evaluating the environmental footprint and is suitable for this study because the prevalent pollutant emitted from electricity generation is $\mathrm{CO}_{2}$. The total energy consumption was calculated by multiplying the mass of each material with the energy intensity of each process (eq.1). The energy intensity of each process was derived from literature [7-10]. The $\mathrm{GWP}_{100}$ was calculated by multiplying the $\mathrm{kg} \mathrm{CO}$ eq produced from the consumption of $1 \mathrm{kWh}$ of electricity with the total energy demands of each process under investigation (eq.2). The $\mathrm{kg} \mathrm{CO}_{2 \text { eq }}$ produced from the consumption of $1 \mathrm{kWh}$ were considered equal to $0,34 \mathrm{~kg} \mathrm{CO}_{2 \mathrm{eq}} / \mathrm{kWh}$ [11].

$$
\begin{array}{r}
E_{i}=m_{i} e_{i} \\
G W P_{100}=E_{i} \frac{m_{\mathrm{CO}_{2} e q}}{k W h}
\end{array}
$$

\subsection{Life Cycle Costing}

A life cycle costing model based on the principles of the Activity Based costing (ABC) method is implemented for evaluating the total cost as well as tracking off the main contributors to the total cost. In $\mathrm{ABC}$ all work steps, with their costs for material, work, etc., are added to build the final cost of a product. This technique demands deep understanding of the process and is able to provide a meticulous insight into the total cost. In the performed LCC, costs associated with labor, material and energy were calculated. The performed cost analysis accounts also for recycling cost which was assumed equal to the energy cost of the recycling process. For calculating the cost and indentify the major cost drivers, Cost Estimation relationships (CERs) were either formulated or adopted from [3]. The CERs are functions that take into account the geometrical features of a material or a product like perimeter, surface, length, shape complexity, mass, as well as the processing parameters for calculating the final cost.

For the purpose of the LCC analysis the cost of $1 \mathrm{kWh}$ was considered equal to 0,114 Euros [12] and the labor cost equal to 32,6 Euros/hour [12]. The cost of the raw material was considered equal to the cost of its constituents. Additionally, the empirical assumption of $80 \%$ of scrap material based on [3] was made for both the RTM and autoclave. The energy cost for both the manufacturing and the recycling stage is calculated as follows:

$$
K_{i}=E_{i} k
$$

where $\mathrm{K}_{\mathrm{i}}$ is the total energy cost, $\mathrm{E}_{\mathrm{i}}$ is the total energy consumption and $\mathrm{k}$ is the cost of 1 $\mathrm{kWh}$ of electricity. 


\section{Results}

\subsection{Life Cycle Analysis}

From the performed environmental analysis the production of raw material (carbon fiber and epoxy) is responsible for $90 \%$ of the total environmental impact (figures 3 and 4). For this reason it is critical to optimize the recycling techniques in order to avoid energy consumption for the production of new carbon fibers.

Producing the canopy involving RTM as the manufacturing process outperfoms autoclaving both in terms of energy consumption (Figures 3a,4a) and Global warming Potential-100 (Figures 3b,4b). This is because of the lower energy intensity demonstrated by the RTM technique. However, despite the environmental credits of this technique, its involvement is expected to lead to a lower product quality (lower mechanical properties, more defects and possibly less complete impregnation) as compared to autoclave.
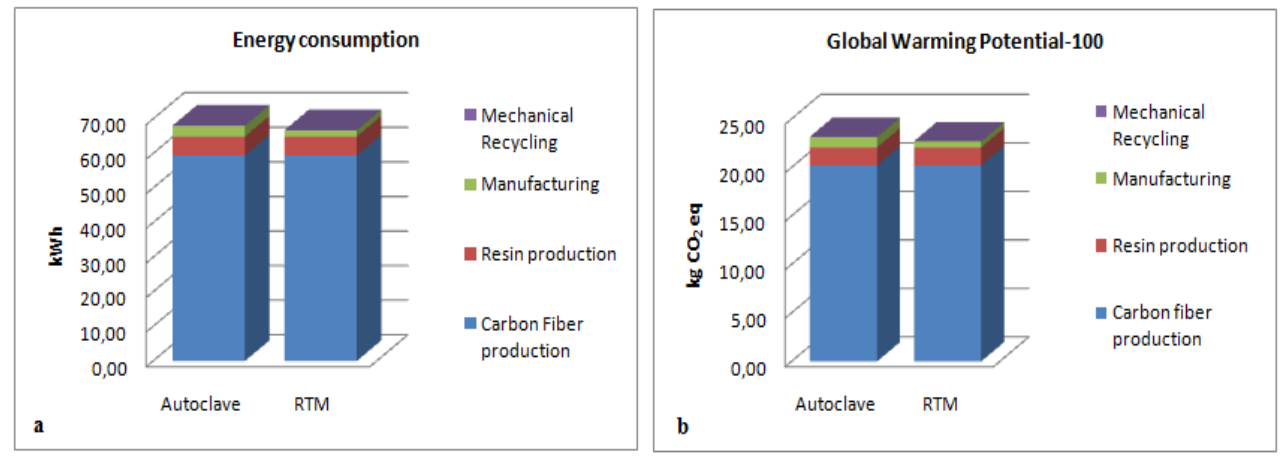

Figure 3: (a) Total energy consumption, (b) Global Warming Potential-100 for each process scenario when mechanical recycling is considered.
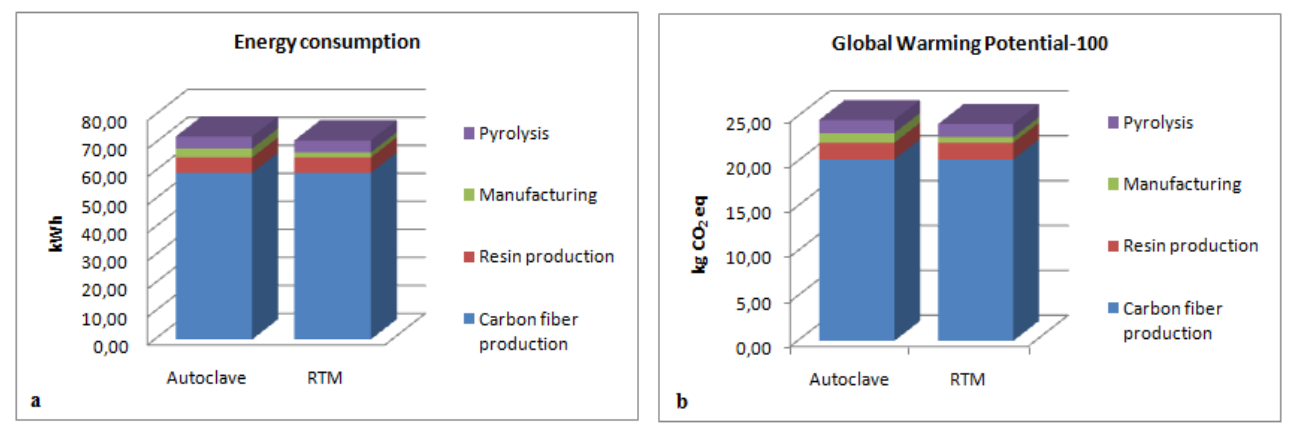

Figure 4: (a) Total energy consumption, (b) Global Warming Potential-100 for each process scenario when pyrolysis is considered. 


\subsection{Life Cycle Costing}

The results from the cost analysis (figure 5a) demonstrated that the main contributors to the total cost are the material and labour costs. The energy as well as the recycling costs occupy a negligible portion of the total cost which is less than $1 \%$. For this reason, only the scenarios where pyrolysis is considered as the recycling technique are shown. Moreover, the more steps needed for preparing the mould and the need for auxiliary materials increases both the labour and the material cost of the autoclave technique up to $29 \%$ (figure 6) and $24 \%$ respectively as compared to RTM. Furthermore, the reduced heating time of RTM leads to a decreased energy cost (figure $5 b$ ).

Figure 7 provides an insight into the effect of the main cost drivers to the total cost. It exhibits the cost variation for storing the component revealing that this activity is more sensitive to a perimeter increase than to a weight increase.
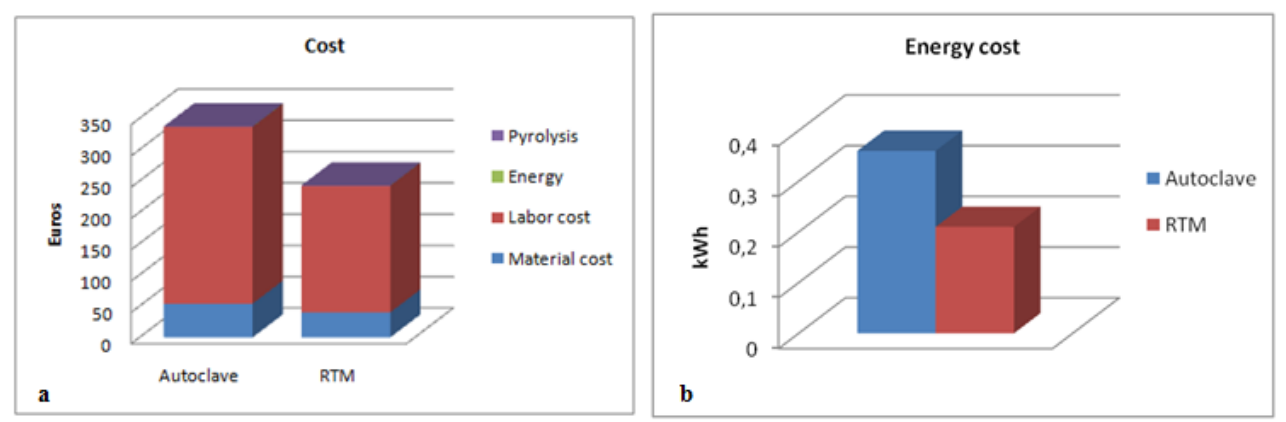

Figure 5: (a) Total LCC for autoclave and RTM, (b) energy cost comparison for autoclave and RTM.
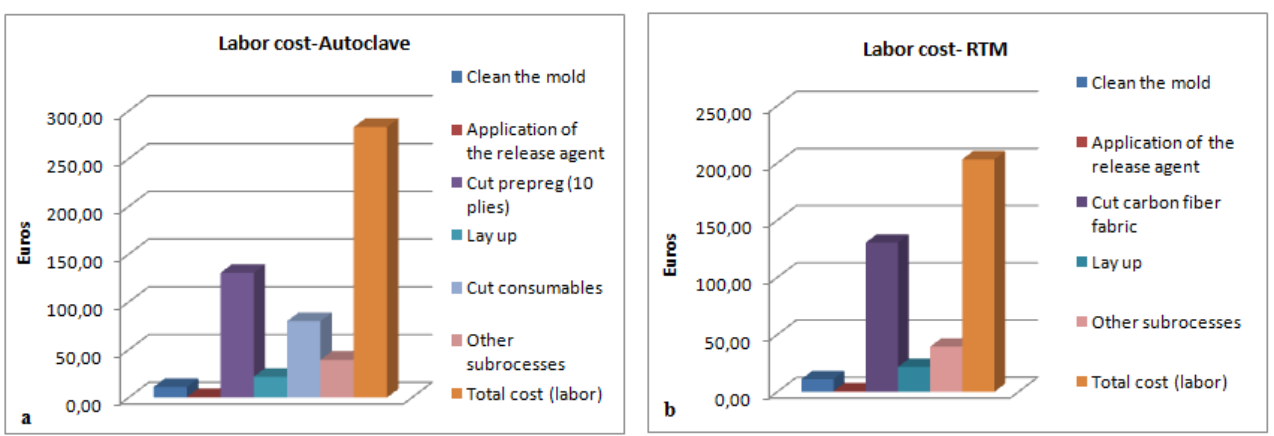

Figure 6: Labor cost breakdown structure for (a) autoclave, (b) RTM. 


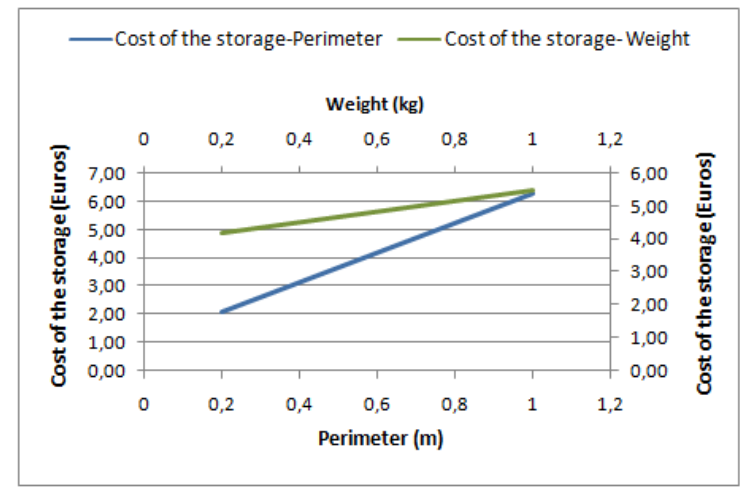

Figure 7: Labor cost for storing the component as a function of weight and perimeter.

\section{Discussion}

In this study Life Cycle analysis and Life Cycle costing models were developed so as quantify the environmental and financial impacts of alternative manufacturing processes (autoclave and RTM) associated with the production of a helicopter's canopy. The canopy was assumed to be made from carbon fiber reinforced thermosetting composite. The results from the analyses demonstrated that RTM outperforms the conventional autoclave technique from an environmental and financial standpoint. The environmental benefits of RTM is the result of the lower energy intensity of the process as compared to autoclaving. Furthermore, the necessity for auxiliary materials (vacuum bag, breather films etc,) needed in autoclaving leads to an increased labor and material cost. On the other hand, autoclaving is still the mainstream process for producing aeronautical components because it provides higher product quality as compared to RTM. Given that quality is of primary concern when producing an aeronautical structural component the above findings underline the need for a trade-off between quality, cost and environmental footprint in the process of optimizing the component and selecting the technique for manufacturing it.

\section{Conclusion}

The environmental and financial performance of two different processing scenarios of the canopy developed in [3] are quantified through the implementation of two broadly applied methods, the LCA and LCC. The results demonstrated the advantage of RTM both in terms of cost and environmental footprint which is the combining result of the lower heating times and the less steps needed for preparing the mold. On the other hand, processing by using the autoclave technique is expected to lead to a higher product quality as compared to the use of RTM. This makes evident the need of a trade-off between different design, manufacturing and recycling options in the process of optimizing a component as well as the necessity of developing tools and concepts making it manageable. 
The present work is a preliminary study performed within NHYTE project [13]. The NHYTE project has received funding from the European Union's Horizon 2020 research and innovation programme under grant agreement No 723309 .

\section{References}

1. A. J. Timmis, A.Hodzic, L. Koh,M. Bonner,C.Soutis, A.W Shafer,L.Dray ,Int. J. Life Cycle Assess., 20, 233-243, (2015).

2. X. Li, R. Bai, and J. McKechnie, J. Clean. Prod., 127, 451-460, (2016).

3. S. G. Pantelakis, C. V. Katsiropoulos, G. N. Labeas, and H. Sibois, Compos. A ,40, 595-606, (2009).

4. Ch.V.Katsiropoulos, G.A.Moraitis, G.N.Labeas, Sp.G.Pantelakis, J. of Plastics Rubber and Composites ,38, 153-161 (2009).

5. F. Weiland, C. Weimer, F. Dumont, Ch. V. Katsiropoulos, Sp. G. Pantelakis, I. Sitaras, A. A. Skordos, E. Berthé, P. de Luca, J. Plastics, Rubber and Composites ,42, 427-436, (2013).

6. ISO 14040, (2006).

7. E. Incorporated, Draft report prepared for the National Renewable Energy Laboratory and U.S Department of Energy, Advanced Manufacturing Office (2016).

8. T. Suzuki and J. Takahashi, Ninth Japan Int. SAMPE Symp., 14-19, (2005).

9. J. Howarth, S. S. R. Mareddy, and P. T. Mativenga, J. Clean. Prod., 81, 46-50, (2014).

10. S. Job, G. Leeke, P. T. Mativenga, G. Oliveux, S. Pickering, and N. A. Shuaib, (2016).

11. www.electricitymap.org.

12. ec.europa.eu.

13. New Hybrid Thermoplastic Composite Aerostructures manufactured by Out of Autoclave Continuous Automated Technologies (NHYTE). EU project, 20172020 . 